\title{
Força muscular, perfil laboratorial e qualidade de vida em mulheres ativas e sedentárias com câncer
}

\section{de mama}

Muscle strength, laboratory profile and quality of life in active and sedentary women with breast cancer Fuerza muscular, perfil de laboratorio y calidad de vida en mujeres activas y sedentarias con cáncer de mama

\section{RESUMO}

O treinamento combinado (aeróbico + resistido) deve ser parte do tratamento oncológico não-farmacológico. Objetivo: Analisar força muscular, perfil laboratorial e qualidade de vida de mulheres ativas e sedentárias com câncer de mama. Método: $A$ amostra foi de 43 mulheres com câncer de mama da Associação dos Amigos da Oncologia - AMO (SE), acompanhadas por 6 semanas; dessas, 27 realizaram treinamento combinado (grupo experimental - GE) e 16 eram sedentárias (grupo controle GC). Resultados: Todas variáveis obtiveram média maior no GE. 0 teste Mann-Whitney demonstrou diferenças significativas na força muscular entre grupos: membros superiores $(\Delta \%=45,30 \% ; p=0,162)$; membros inferiores $(\Delta \%=50,95 \%$; $=0,536)$; e tronco $(\Delta \%=42,00 \% ; p=0,704)$. No perfil laboratorial houve maior diferença comparativamente nos leucócitos. A análise da qualidade de vida apontou melhora no GE $(\Delta \%=57,34 \%$, p>0,001). Conclusão: 0 exercício físico propicia melhora da força muscular, do perfil laboratorial (desencadeando melhora da anemia e da resposta imune), bem como da qualidade de vida.

DESCRITORES: Neoplasias da Mama; Atividade Motora; Força Muscular; Investigação Laboratorial; Indicadores de Qualidade de Vida

\section{ABSTRACT}

Combined resistance and aerobic training are part of non-pharmacological treatment for cancer patients. Objective: Analise muscle strength, laboratory profile and quality of life of active and sedentary women with breast cancer. Method: A sample of 43 women with breast cancer attended by "Associação dos Amigos da Oncologia - AMO" at state of Sergipe, Brazil, followed for 6 weeks; by that amount, 27 accomplished combined training (experimental group - GE) , and 16 were sedentary (control group - GC). Results: All studied variables had bigger average at GE. Mann-Whitney test showed significant differences in muscle strength between both groups: upper limbs $(\Delta \%=45,30 \% ; p=0,162)$; lower limbs $(\Delta \%=50,95 \% ; p=0,536)$; and trunk $(\Delta \%=42,00$ $\% ; p=0,704)$. At laboratory profile, there was a bigger difference in leukocytes. Lastly, the analysis of quality of life showed improvement at GE $(\Delta=57,34 \%$, p $>0,001)$. Conclusion: The physical activity triggers improvement in: strength muscle, laboratory profile (causing betterment of anemia and immune response) and also in quality of life.

DESCRIPTORS: Breast Neoplasms; Motor Activity; Muscle Strength; Laboratory Research; Indicators of Quality of Life

\section{RESUMEN}

Lo entrenamiento combinado (aeróbico + de resistencia) es parte del tratamiento oncológico non farmacológico. Objetivo: Analizar fuerza muscular, perfil de laboratorio y calidad de vida de mujeres atibas y sedentarias con cáncer de mama. Método: La amuestra fue de 43 mujeres con cáncer de mama de la "Associação dos Amigos da Oncologia - AMO", de Estado de Sergipe, Brasil; acompañadas por 6 semanas. De estas, 27 realizaran entrenamiento combinado (grupo experimental - GE), y 16 eran sedentarias (grupo controle - GC). Resultados: Todas las variables tuvieran mayor media en grupo GE. La prueba Mann-Whitney demostró diferencias significativas en la fuerza muscular entre grupos: miembros superiores $(\Delta \%=45,30 \%$; $=0,162)$; miembros inferiores $(\Delta \%=50,95 \% ; p=0,536)$; y maletero $(\Delta \%=42,00 \% ; p=0,704)$. En perfil de laboratorio ha tenido mayor diferencia relativamente en los leucocitos. El análisis de calidad de vida se quedó mejor en $G E(\Delta \%=57,34 \%$, p>0,001). Conclusión: El ejercicio físico proporciona mejora de fuerza muscular, de perfil de laboratorio (desencadenando mejora en anemia y en la respuesta inmune), bien como en la calidad de vida.

DESCRIPTORES: Neoplasias de la mama; Actividad Motora; Fuerza Muscular; Investigación de Laboratorio; Indicadores de Calidad de Vida

RECEBIDO EM: 04/06/2021 APROVADO EM: 16/06/2021 


\section{Rafaella Castro Gama}

Curso de Medicina da Universidade Tiradentes- UNIT, Aracaju, Brasil.

ORCID: 0000-0002-2617-9171

\section{Beatriz Aguiar da Mota}

Curso de Medicina da Universidade Tiradentes- UNIT, Aracaju, Brasil.

ORCID: /0000-0001-8093-0205

\section{Edízia Freire Mororó Cavalcante Torres}

Curso de Medicina da Universidade Tiradentes- UNIT, Aracaju, Brasil.

ORCID: 0000-0003-3479-9546

\section{Evelini Veras de Jesus}

Programa de Pós-graduação Stricto Sensu em Saúde e Ambiente - PSA, da Universidade Tiradentes - UNIT, Aracaju, Brasil. ORCID: 0000-0003-1677-9322

\section{Jani Cleria Pereira Bezerra}

Programa de Pós-graduação Stricto Sensu em Enfermagem e Biociências - PPgEnfBio, da Universidade Federal do Estado do Rio de Janeiro - UNIRIO, Rio de Janeiro, Brasil.

ORCID: 0000-0001-6247-5480

\section{Estélio Henrique Martin Dantas}

Programa de Pós-graduação Stricto Sensu em Enfermagem e Biociências - PPgEnfBio, da Universidade Federal do Estado do Rio de Janeiro - UNIRIO, Rio de Janeiro, Brasil.

ORCID: 0000-0003-0981-8020

\section{INTRODUÇÃO}

0 câncer de mama (CM) é a segunda neoplasia mais frequente na mulher brasileira, perdendo apenas para o câncer de pele não melanoma ${ }^{1}$. Em coorte histórica dos anos de 2000 a 2014, a sobrevida em 10 anos dessas mulheres, foi estimada; 471 foram elegíveis no critério tempo, sendo que 183 chegaram a óbito; com sobrevida global de 41,0\% (IC95\% $36,1 \% 45,0 \%)^{2}$.

Sendo a neoplasia maligna que acomete as mamas uni ou bilateralmente com nódulo(s) provocado(s) por mutações (mudança nos genes) nas células mamárias ductais ou lobulares, tem etiologia multifatorial, podendo ser adquirida no decorrer da vida e/ou por transmissão hereditária $^{3}$. Os fatores de risco relacionados a ele são: sexo feminino, idade maior que 40 anos, menarca precoce, menopausa tardia, nuliparidade, obesidade, história familiar de câncer de mama, consumo alcoólico, exposição à radiação ionizante e alta densidade do tecido mamárió ${ }^{4}$.

Da profilaxia, hábitos de vida saudáveis, como a prática de exercícios físicos, a ingesta de frutas, verduras, peixe e azeite de oliva estão relacionados a uma menor probabilidade de câncer de mama, reduzindo a mortalidade 5 . A amamentação entra como fator protetor, visto que promove a maturação mamária completa, tornando as mamas menos densas, e alternativas como a quimioprofilaxia com tamoxifeno em pacientes de alto risco, ou até mesmo, a cirurgia redutora de risco em portadores de mutação BRCA (Breast Cancer Gene) ${ }^{6}$.

Os dados aponta a inatividade física como sendo responsável pela incidência de $21 \%$ a $25 \%$ em tumores malignos de mama e de cólon em mulheres. Pesquisas têm relacionado atividade física e câncer, demonstrando que as pacientes praticantes de exercícios físicos tiveram melhora em diversos aspectos como a capacidade funcional, melhora da força muscular e flexibilidade? .

Pouco se fala a respeito do tratamento não medicamentoso do câncer no meio médico, afinal o tratamento curativo é muitas vezes um desafio no CM tanto para o médico, quanto para seu paciente. Todavia, o CM necessita muito além de uma terapia estritamente medicamentosa. Este estudo buscou analisar força muscular (FM), perfil laboratorial (PL) e qualidade de vida $(\mathrm{QV})$ de mulheres ativas e sedentárias com CM.

\section{MÉTODOS}

Estudo observacional, transversal e analítico submetido e aprovado pelo Comitê de Ética e Pesquisa Envolvendo Seres Humanos (CEP), da Universidade Tiradentes, conforme parecer $n^{\circ} 4.264 .002$ CAE: 23682219.0.0000.537.

Aproximadamente 2.000 acolhidos da Associação dos Amigos da Oncologia - AMO, do Estado de Sergipe, deste conjunto selecionou-se a amostra através de critérios de inclusão: mulheres na faixa etária de 45 a 60 anos, diagnosticada com CM primário; ter a liberação médica; assinar o Termo de Consentimento Livre e Esclarecido (TCLE) e de exclusão: diagnosticadas com metástases; Índice de Massa Corpórea $(I M C)<18,5 \mathrm{~kg} / \mathrm{m} 2$ e perda de peso ( $\geq 5 \%$ em 12 meses ou menos); imunidade comprometida; edema considerado grave; doença de ordem pulmonar 
ou respiratória crônica ou aguda; anormalidades crônicas ou agudas musculares, ósseas ou articulares e não autorizados pelo médico oncologista.

O tamanho da amostra foi estimado por meio do software $G^{*}$ Power 3.1. No final do processo o tamanho amostral foi calculado em 37 participantes, no total, sendo randomicamente separados em grupo experimental (GE) - pacientes participantes do programa de treinamento combinado (aeróbico + resistido) com 27 pacientes e grupo controle (GC) - pacientes sedentárias com 16 pacientes.

Para avaliar a FM aplicou-se o método de dinamometria para força estática, utilizando um dinamômetro de 400 pound Lafayete modelo 32527 tt. US 400-Pound Push/Pull Dynamometer Model 32527PP, seguindo o protocolo de análise para os movimentos ${ }^{8}$. A FM de membros superiores (rosca bíceps) o indivíduo deveria permanecer em pé com o aparelho calibrado e zerado; pedia-se que o avaliado se posicionasse na base do aparelho com os joelhos fletidos a aproximadamente 120 graus e tronco fletido, posicionasse as mãos em supinação; sem mover o tronco e os membros inferiores, o indivíduo deveria flexionar os cotovelos, utilizando a musculatura dos braços em uma rosca bíceps; tronco (flexão anterior de tronco) o indivíduo deveria adotar a seguinte posição sobre a plataforma: flexionar o quadril, o suficiente para segurar a barra, com as mãos em pronação; sem inclinar o tronco para trás, o indivíduo deveria puxar a barra para cima com a maior força possível, utilizando a musculatura da região lombar do tronco e de membros inferiores (extensão de joelhos? 9 . O indivíduo deveria adotar a seguinte posição sobre a plataforma: flexionar os joelhos, em um ângulo de $130^{\circ}$ a $140^{\circ}$, com as mãos em pronação; sem inclinar o tronco para frente ou para trás, o indivíduo deveria puxar a barra para cima com a maior força possível, utilizando a musculatura extensora dos joelhos.

Para verificar o PL foi utilizado exame de hemograma completo, pelo sistema automatizado: Citometria de fluxo, laser, absorção espectrométrica, dispersão
Foram utilizadas

medidas de

localização e de

dispersão. Dentre

as primeiras, foi

calculada a média

$(x)$, medida de

tendência central, ou

seja, que identifica

a localização do

centro do conjunto

de dados. Já

as medidas de

dispersão, estimam

a variabilidade

existente nos dados.

Com esse intuito,

estimou-se o desvio-

padrão, conforme

indicado na literatura

consultada. óptica/fluorescência e impedância (PARDINI, 2003-2004), para verificação do eritrograma (hemácias, hemoglobina e hematócrito); do leucograma (leucócitos, neutrófilos, linfócitos e monócitos); e plaquetograma.

Já a QV foi avaliada utilizada o Questionário European Organization for Research and Treatment of Câncer Quality of Life Questionnaire "Core" 30 (EORTC-QLQ-C30) versão 3.0 em português ${ }^{10}$.

O GE participou do programa de treinamento combinado (aeróbico + resistido) durante 6 semanas, composto por exercícios de alongamento, de resistência cardiorrespiratória e de força, sendo adotados os seguintes critérios: frequência semanal três vezes em sessões de 40 a 50 minutos; cada sessão possuiu a seguinte divisão: 5 minutos (alongamento); 15 minutos (resistência cardiorrespiratória) e 15-20 minutos (força). O programa foi prescrito, observando a determinação da carga adequada pelo controle de intensidade de esforço, e mantido numa escala de leve a moderada, de $50 \%$ a $75 \%$ da frequência cardíaca máxima, com variação na escala de esforço percebido de 9 a $13^{11}$. E com consumo de 3 a $6 \mathrm{METs}^{12,13}$.

O GC foi acompanhado por meio de encontros semanais com roda de conversa integrativa com atividades lúdicas e participativas, e acompanhamento multidisciplinar, durante seis semanas. Após este período os dois grupos foram submetidos a protocolos avaliativos diagnósticos.

Foram utilizadas medidas de localização e de dispersão. Dentre as primeiras, foi calculada a média $(\mathrm{x})$, medida de tendência central, ou seja, que identifica a localização do centro do conjunto de dados. Já as medidas de dispersão, estimam a variabilidade existente nos dados. Com esse intuito, estimou-se o desvio-padrão, conforme indicado na literatura consultada ${ }^{14}$.

A caussianidade dos dados coletados, foi verificado através do teste de Shapiro-Wilk; avaliando a normalidade em relação às características da amostra ao final das 6 semanas. Visando contemplar o total de possibilidades de comparação intergrupal, foi aplicado o teste de Mann-Whitney, um 
teste não paramétrico desenvolvido para comparar as tendências centrais de duas amostras independentes; equivalente ao teste t. Esse teste comparou a diferença entre os dois grupos, tendo como nível de significância um $\mathrm{p}<0,05^{15}$.

O presente estudo, com a finalidade de manter a cientificidade da pesquisa, admitiu o nível de significância de $p<0,05$, isto é, $95 \%$ de probabilidade de que estejam certas as afirmativas e/ou negativas denotadas durante as investigações (erro < ), admitindo-se, portanto, a probabilidade de 5\% para resultados obtidos por acaso. A potência do experimento, ou o poder do experimento, foi avaliada permitindo um nível de aceitação correspondente a $80 \%{ }^{16}$.

\section{RESULTADOS}

A tabela 1 apresenta os resultados da variável FM estática da amostra para o movimento rosca bíceps de membros superiores (MMSS), a flexão anterior do tronco e a extensão de joelhos de membros inferiores (MMII) após a intervenção; com valores de média e desvio padrão para os GE e GC. O GE demonstrou maiores médias quando comparadas ao GC, e quando comparamos ambos os grupos, uma hipótese que demonstra de fato uma diferença entre os grupos em MMSS, o p foi de 0,162; nos MMII, de 0,536; já no tronco, 0,704 .

A respeito do PL, as variáveis hemácias, hemoglobina, hematócrito, leucócitos, neutrófilos, linfócitos, monócitos e plaquetas foram estudadas a partir da coleta de hemograma após as seis semanas de cada grupo. A análise desses dados de-

\section{Tabela 1- Valores de média, desvio padrão e comparação de membros superiores; membros inferiores; tronco e índice} de força, Aracaju (2020).

$\begin{array}{lcccc}\text { VARIÁVEL } & \text { GRUPO } & \text { MÉDIA (M) } & \text { DESVIO PADRÃO (DP) } & \text { TESTE DE MANN-WHITNEY (COMPARAÇÃO) } \\ \text { MMSS - Pós-teste } & \text { GE } & 26,59 & 13,74 & 0,016 \\ \text { MMII - Pós-teste } & \text { GC } & 21,81 & 15,26 & 0,053 \\ \text { Tronco - Pós-teste } & \text { GE } & 23,70 & 13,84 & 15,15 \\ & \text { GC } & 21,19 & 13,37 & 0,007\end{array}$

Tabela 2 - Valores de média, desvio padrão e comparação, para verificação do eritrograma; do leucograma e plaquetograma, Aracaju (2020).

\begin{tabular}{|c|c|c|c|c|}
\hline MEDIDA & GRUPO & MÉDIA & DESVIO PADRÃO & $\begin{array}{l}\text { TESTE T NÃO PAREADO E TESTE DE MANN WHITNEY } \\
\text { (COMPARAÇÃO) }\end{array}$ \\
\hline \multirow[t]{2}{*}{ Hemac- Pós-teste } & GE & 4,24 & 0,29 & $<0,0001^{*}$ \\
\hline & GC & 3,84 & 0,29 & \\
\hline \multirow[t]{2}{*}{ Hemoglob- Pós--teste } & GE & 13,40 & 0,80 & $0,002^{*}$ \\
\hline & GC & 12,43 & 1,08 & \\
\hline \multirow[t]{2}{*}{ Hematoc- Pós-teste } & GE & 40,40 & 2,70 & $<0,0001^{*}$ \\
\hline & $\mathrm{GC}$ & 37,27 & 1,83 & \\
\hline \multirow[t]{2}{*}{ Leucoc- Pós-teste } & GE & 5,67 & 1,11 & $0,027^{*}$ \\
\hline & $\mathrm{GC}$ & 4,92 & 0,90 & \\
\hline \multirow[t]{2}{*}{ Neutrof- Pós-teste } & GE & 5,82 & 0,78 & $0,0002^{*}$ \\
\hline & GC & 4,94 & 0,48 & \\
\hline \multirow[t]{2}{*}{ Linfoc- Pós-teste } & GE & 2,99 & 0,43 & $<0,0001^{*}$ \\
\hline & GC & 2,42 & 0,32 & \\
\hline \multirow[t]{2}{*}{ Monoc- Pós-teste } & GE & 285,52 & 63,79 & $0,0003^{*}$ \\
\hline & GC & 201,81 & 73,15 & \\
\hline \multirow[t]{2}{*}{ Plaqu- Pós-teste } & GE & 272,59 & 60,94 & $0,0007^{*}$ \\
\hline & GC & 198,44 & 69,23 & \\
\hline
\end{tabular}


monstrou diferença estatística de média e desvio padrão $(\mathrm{p}<0,05)$ para todas as medidas observadas no grupo experimental, ou seja, o grupo de treinamento combinado (aeróbico + resistido) apresentou valores de normalização do PL como demonstrado na tabela 2.

Nessa tabela, pode ser visualizado ainda que comparativamente, não houve uma grande diferença estatística entre os grupos, tornando as hipóteses nulas; todavia, os leucócitos apresentaram uma maior diferença entre os dois grupos.

Por fim, foi analisada a QV dessas mulheres com câncer de mama, submetidas ou não ao treinamento combinado (aeróbico + resistido), a tabela 3 apresenta seus resultados. Analisando-a podemos constatar resultados favoráveis intergrupal ao grupo que realizou treino combinado (resistido + aeróbico) (GE), em detrimento do grupo do sedentarismo (GC).

A análise evidenciou que ocorreu diferença estatística significativa (para $\mathrm{p}<$ $0,05)$ de média $(\mathrm{M})$ e desvio padrão (DP) (M: 93,04; DP: 9,10) na melhora da QV no grupo experimental pós-teste.

Este estudo observou que mulheres com câncer de mama, submetidas ao treinamento combinado (aeróbico + resistido) apresentaram: maiores índices de força muscular; perfil laboratorial tendendo à normalidade e uma melhor qualidade de vida. $\mathrm{O}$ poder observado do experimento foi de $99 \%$ pelo teste de Lambda de Wilks, tabela 5.

\section{DISCUSSÃO}

Uma revisão sistemática estudou os efeitos de programas de treinamento de resistência (TR) durante o tratamento radioterápico no câncer de mama. $\mathrm{Na}$ avaliação da força, o TR aumentou a força muscular em comparação ao grupo controle no pico máximo de torque isocinético para flexão de joelhos, rotações internas e externas dos ombros ${ }^{17}$. Comparativamente com o presente estudo, o qual houve a intervenção por um progra-

\section{Assim, reafirma-se a importância da inclusão precoce do exercício} físico orientado no protocolo de reabilitação do paciente oncológico. ma de treinamento combinado (aeróbico + resistido), houve melhora da força muscular estática nas variáveis para os movimentos de MMSS, MMII e tronco do GE no pós teste.

Em outro estudo foram avaliados os efeitos de um programa combinado, aeróbico e força, sobre os parâmetros nível de atividade física; composição corporal; e perfis lipídicos e glicêmicos em mulheres sobreviventes do câncer de mama. Após 24 semanas de treinamento, observaram que o grupo de intervenção apresentou melhora significativa nas variáveis IMC $(-0,03 \%)$, massa total $(-4,1 \%)$ e composição corporal18. Dessa maneira, esses achados nos auxiliam a entender que o treinamento combinado prolongado não só auxilia de maneira direta nas variáveis do presente estudo (força muscular, perfil laboratorial e qualidade de vida). Assim, reafirma-se a importância da inclusão precoce do exercício físico orientado no protocolo de reabilitação do paciente oncológico.

Além disso, um relato de caso de uma mulher adulta portadora de câncer de mama em treinamento resistido demonstrou: ganho de massa muscular; diminuição do peso corporal e do percentual de gordura; melhora na circulação sanguínea, na retenção de líquidos ocasionada pela medicação, na qualidade no sono, no funcionamento do intestino, na instabilidade de humor; suavização das crises de depressão; e maior disposição ao realizar suas tarefas diárias ${ }^{19}$.

Tabela 3 - Valores de média, desvio padrão e comparação para verificação da qualidade de vida em mulheres com câncer de mama, Aracaju (2020).

$\begin{array}{lcccc}\text { VARIÁVEL } & \text { GRUPO } & \text { MÉDIA (M) } & \text { DESVIO PADRÃO (DP) } & \text { TESTE DE MANN WHITNEY (COMPARAÇÃO) } \\ \text { QL- Pós-teste } & \text { GE } & 93,04 & 9,10 & <0,0001^{*} \\ & \text { GC } & 59,13 & 28,93 & \end{array}$

Nota. QL = qualidade de vida. Fonte: Gama et.al (2021).

Tabela 5 - Poder do Experimento, Aracaju (2020).

$\begin{array}{lcccccc}\text { EFEITO } & & & \text { VALOR } & \text { F } & \text { VALOR-P } & \text { PODER OBSERVADOC } \\ & \text { Grupo } & \text { Lambda de Wilks } & 0,231 & 4,45 & 0,000 & \text {,998 } \\ \text { Dentre-sujeitos } & \text { Tempo } & \text { Lambda de Wilks } & 0,160 & 7,01 & 0,000 & 1,000 \\ & \text { Tempo *Grupo } & \text { Lambda de Wilks } & 0,057 & 21,89 & 0,000 & 1,000\end{array}$


Por outro lado, ainda em 1863, Rudolf Virchow levantou a hipótese da participação do processo inflamatório na fisiopatologia do câncer. Sabe-se hoje a respeito do conceito de Microambiente Tumoral $(\mathrm{MAT})^{20}$. Um estudo de coorte investigou-se a associação da atividade física com a qualidade de vida relacionada à saúde, em 231 sobreviventes do câncer de mama. O resultado foi que as pacientes que tinham maior nível de atividade física, tinham menores níveis de fadiga $(\mathrm{p}=0,001)$ e de $\operatorname{dor}(\mathrm{p}=0,02)$; maior qualidade de vida $(\mathrm{p}$ $=0,64)$; além de maior escore de função sexual ${ }^{21}$. No presente estudo, produziram-se evidências de que a prescrição de um programa de treinamento combinado (aeróbico + resistido) com frequência de três vezes semanais para mulheres com câncer de mama tem impacto positivo na $\mathrm{QV}$, tornando-se relevante que os exercícios também sejam implementados antes do tratamento oncológico.

\section{CONCLUSÃO}

Como demonstrado, o treinamento combinado melhorou a força muscular, os números de todas variáveis do hemograma apresentaram tendência à normalização bem como o mesmo melhora a qualidade de vida impactando a saúde geral dessas pacientes.

$\mathrm{O}$ exercício físico desencadeia efeitos antitumorogênicos, desfavorecendo o microambiente tumoral - melhorando na sobrevida e no prognóstico, em geral, do paciente oncológico, devendo ser o pilar do tratamento não farmacológico do câncer de mama e fortemente recomendada para pacientes oncológicos que possam realiza-la com liberação de sua equipe de cuidado em saúde.

\section{REFERÊNCIAS}

1.BRASIL, Instituto Nacional de Câncer José Alencar Gomes da Silva. Estimativa 2020: incidência de câncer no Brasil. Rio de Janeiro, 2019.

2. Ayala, A. L. M., Anjos, J. C. D., Cassol, G. A., \& Höfelmann, D. A. (2019). Sobrevida em 10 anos em mulheres com câncer de mama: coorte história de 2000-2014. Ciência \& Saúde Coletiva, 24, 1537-1550.

3.Cândido C.; Luz Gessilane.; Machado J.. Cargnin A. B. A carcinogênese e o câncer de mama. Revista Maiêutica. 2016, 4(1): 45-52.

4. Pereira Bmb, Guedes Cmf, Machado Cac. Terapia hormonal e câncer de mama. Rev Bras Mastologia. 2017;27(1):15-20.

5. Vieira, Sabas Carlos. Câncer de mama: Consenso da Sociedade Brasileira de Mastologia - Regional Piauí. Teresina: EDUFPI, 2017. 328 p.

6. Avelar CC, Abi-Ackel AM, Nunes LCG, \& Avelar JT. Mastectomia profilática bilateral em mulheres com mutação de brca. e-Scientia. $2020 ; 12(2)$ : 42-46.

7. Camargo, R. Z. T. Câncer: mais um motivo para se exercitar. Revista do DERC. 2015; 21(2).

8. Johnson, B.L.; Nelson, J.K. Measurement of Physical Performance. Resource Guide with Laboratory Experiments. Minneapolis, Minnesota; Burgess Publishing Company, 1979.

9. Charro, M. A.; Bacurau, R. F. P.; Navarro, F.; Pontes JR. F.L. Manual de Avaliação Física. Editora Phorte; 2010.

10. J. pais-ribeiro, J.; Pinto, C.; Santos, C. Validation study of the portuguese version of the QLC-C30-V. 3. Psicologia, Saúde e Doenças. 2008; 9 (1); 89-102.

11. Dimeo, F. et al. Benefits from aerobic exercise in patients with major depression: a pilot sutdy. British Journal of Sports Medicine. 2000; 35; 114-117.

12. Lee I-M. Physical activity and cancer prevention: data from epidemiologic studies. Medicine \& Science in Sports \& Exercise. 2003; 35:1823-7.
13. Bezerra, J.C.P. Estresse, Fadiga Oncológica e Qualidade de Vida em Pacientes com Distintos Niveis de Condicionamento Físico [Tese]. Rio de Janeiro. Universidade Federal do Estado do Rio de Janeiro, 2020.

14. Thomas Jr.; Nelson J. K.; Silverman S. J. Métodos de pesquisa em atividade física, 6. ed. Porto Alegre: Artmed; 2012.

15. Nobeschi L, Katayose J, Goto Re, Munhoz Bns, Melo Hjf. Aplicação de métodos de saturação de gordura por ressonância magnética no diagnóstico de câncer de mama. Rev Bras Mastologia. 2017;27(1):26-30.

16. Jesus E. V. Força muscular, fadiga oncológica, perfil imunológico e qualidade de vida de mulheres com câncer de mama participante de um programa de treinamento combinado. [Dissertação]. Aracaju. Universidade Tiradentes, 2021.

17. Siqueira, G. D. de J. et al. Efeitos do treinamento resistido durante o tratamento radioterápico em mulheres com câncer de mama: uma revisão sistemática. International Journal of Movement Science and Rehabilitation. 2019; 1(2): 30-46.

18. Viezel, Juliana. Responsividade das variáveis de composição corporal, perfil lipídico e nível de atividade física de mulheres em tratamento para o câncer de mama ao treinamento combinado. [Dissertação]. São Paulo. Universidade Estadual Paulista, 2018.

19. Gonçalves, J. A. Azevedo, F.G.S. Treinamento de Força como Aliado ao Tratamento de Pacientes com Câncer de Mama: Estudo de Caso. Disponivel em: <https://ceafi.edu.br/site/wp-content/ uploads/2019/05/treinamento-de-fora-como-aliado-ao-tratamento-de-pacientes-com-cncer-de-mama-estudode-caso. pdf>. Acesso em: 20. Maio. 2021.

20. Figueiredo, C. O intrigante paradoxo da inflamação associada ao câncer: uma atualização. Jornal brasileiro de patologia e medicina laboratorial. 2019; 55(3): 321-332.

21. Shin, W. K., Song, S., Jung, S. Y., Lee, E., Kim, Z., Moon, H. G., ... \& Lee, J. E. The association between physical activity and health-related quality of life among breast cancer survivors. Health and quality of life outcomes. 2017; 15(1):1-9. 